Probability, Networks and Algorithms

A tandem queue with Lévy input: a new representation of the downstream queue length

K.G. Dẹbicki, M.R.H. Mandjes, M.J.G. van Uitert

Report PNA-e0603 January 2006 
Centrum voor Wiskunde en Informatica (CWI) is the national research institute for Mathematics and Computer Science. It is sponsored by the Netherlands Organisation for Scientific Research (NWO).

CWI is a founding member of ERCIM, the European Research Consortium for Informatics and Mathematics.

CWI's research has a theme-oriented structure and is grouped into four clusters. Listed below are the names of the clusters and in parentheses their acronyms.

\section{Probability, Networks and Algorithms (PNA)}

Software Engineering (SEN)

Modelling, Analysis and Simulation (MAS)

Information Systems (INS)

Copyright (C) 2006, Stichting Centrum voor Wiskunde en Informatica

P.O. Box 94079, 1090 GB Amsterdam (NL)

Kruislaan 413, 1098 SJ Amsterdam (NL)

Telephone +31205929333

Telefax +31205924199

ISSN 1386-3711 


\title{
A tandem queue with Lévy input: a new representation of the downstream queue length
}

\begin{abstract}
In this paper we present a new representation for the steady state distribution of the workload of the second queue in a two-node tandem network. It involves the difference of two suprema over two adjacent intervals. In case of spectrally-positive Lévy input, this enables us to derive the Laplace transform and Pollaczek-Khintchine representation of the workload of the second queue. Additionally we obtain the exact distribution of the workload in the case of Brownian and Poisson input, as well as some insightful formulas representing the exact asymptotics for alphastable Lévy inputs.
\end{abstract}

2000 Mathematics Subject Classification: 60G51, 60K25

Keywords and Phrases: large-buffer asymptotics, stationary independent increments, tandem networks, Laplace transform, Lévy processes, communication networks

Note: This paper will appear in "Probability in the Engineering and Informational Sciences" 



\title{
A tandem queue with Lévy input: a new representation of the downstream queue length
}

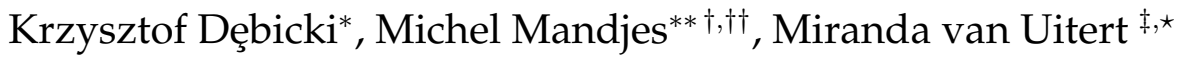 \\ * Mathematical Institute, University of Wrocław \\ pl. Grunwaldzki 2/4, 50-384 Wrocław, Poland \\ ${ }^{* *}$ CWI, P.O. Box 94079, 1090 GB Amsterdam, the Netherlands \\ ${ }^{\dagger}$ Korteweg-de Vries Institute, University of Amsterdam
}

Plantage Muidergracht 24, 1018 TV Amsterdam, Amsterdam, the Netherlands

${ }^{\dagger \dagger}$ EURANDOM, P.O. Box 513, 5600 MB Eindhoven, the Netherlands

${ }^{\ddagger}$ The Netherlands Cancer Institute

Plesmanlaan 121, 1066 CX Amsterdam, the Netherlands

* Information and Communication Theory Group, Delft University of Technology

P.O. Box 5031, 2600 GA Delft, the Netherlands

\begin{abstract}
In this paper we present a new representation for the steady state distribution of the workload of the second queue in a two-node tandem network. It involves the difference of two suprema over two adjacent intervals. In case of spectrally-positive Lévy input, this enables us to derive the Laplace transform and Pollaczek-Khintchine representation of the workload of the second queue. Additionally we obtain the exact distribution of the workload in the case of Brownian and Poisson input, as well as some insightful formulas representing the exact asymptotics for $\alpha$-stable Lévy inputs.
\end{abstract}

Key words: large-buffer asymptotics - stationary independent increments - tandem networks - Laplace transform - Lévy processes - communication networks

2000 Mathematics Subject Classification: 60G51 (primary), 60K25 (secondary).

Email: debicki@math.uni.wroc.pl, michel@cwi.nl, m.uitert@nki.nl 


\section{Introduction}

Traffic engineering greatly benefits from models that are capable of accurately describing and predicting the performance of the system. The network nodes are usually modeled as queues, and queueing theory can be used to analyze the performance (in terms of loss, delay, throughput, etc.) of these nodes. However, most studies address performance issues for single nodes, which is evidently an oversimplification of reality. This justifies the growing interest in the performance of traffic streams traversing concatenations of queues. Measurement studies have provided empirical evidence that in particular cases network traffic can be approximated by Gaussian processes, e.g., Brownian motion or fractional Brownian motion (fBm). Mikosch et al. [27] and Kaj and Taqqu [22] have focused on the cases in which it is not accurate to assume Gaussianity; in [27] it is argued that, if the rate at which connections are initiated is modest relative to the tail of the connectionlength distribution (which is assumed regularly varying), it is preferred to approximate the traffic stream by a stable Lévy motion. More justification for the use of Lévy motions in a communication networking context can be found in, e.g., [10].

This paper focuses on a two-node tandem network with Lévy input - we note, however, that it is straightforward to extend the analysis to tandem networks with more than two nodes. Our purpose is to analyze the buffer content distribution of the downstream (i.e., second) queue. Some results are known for special cases of this model. The tandem queue with Poisson arrivals, for instance, was analyzed in detail, but a considerable part of the results is in terms of Laplace transforms; see, e.g., [33, 37, 41]. For Brownian motion input the exponential decay rate of the queue-size distribution of the second queue is given in, e.g., [26].

Our paper is related to earlier works by Kella and Whitt [25] and Kella [23]. These papers also consider tandem queues with Lévy input, but there it is required that the input is non-decreasing. This assumption, however, excludes classes of input processes such as Brownian motion and $\alpha$-stable Lévy motions (with $\alpha<2$ ). The approach taken in this paper covers these important cases. Moreover, in [25] and [23] the emphasis is on moments and Laplace transforms, whereas a substantial part of our results is in terms of explicit expressions for the probability distribution of the downstream queue (or its tail behavior).

This paper is organized as follows. Section 2 finds a remarkable new representation of the queue size of the downstream queue. The new formula holds for general (not only Lévy) input processes and involves the difference of two suprema over two adjacent intervals. The derivation relates to results of [26]. The specification of this representation for spectrally-positive Lévy input enables us to analyze (in Section 3) the down- 
stream queue in terms of Laplace transforms. This leads to the analogue of the result by Zolotarev [42] for the single queue. Additionally we derive the Pollaczek-Khintchine representation of the distribution of the downstream queue that extends Theorem 4.1 in [25] from 'subordinator Lévy input' to spectrally-positive Lévy input.

Section 4 applies the general results to a number of examples with light-tailed input. For both Brownian and Poisson input we give the exact distribution of the workload of the second queue. Our analysis indicates that in this light-tailed case there are two regimes: there is a critical service rate $c_{1}^{\star}$ such that (i) for $c_{1}>c_{1}^{\star}$ all traffic entering the first queue is essentially served immediately, and goes directly into the second queue, such that traffic is not 'reshaped' by the first queue; (ii) for $c_{1} \leq c_{1}^{\star}$, the first queue does play a role in delaying and reshaping the traffic before entering the second queue. This behavior is in line with earlier results in, e.g., [11, 26].

Section 5 deals with examples featuring heavy-tailed input. We find asymptotics and bounds of the tail distribution when the input is $\alpha$-stable Lévy motion and compound Poisson (with regularly-varying jumps).

\section{Model and fundamental result}

In this section we first describe our tandem queueing model. Then we present our fundamental result.

\subsection{Model}

In this paper we consider a two-node tandem network with service rate $c_{1}$ at the first node, and service rate $c_{2}<c_{1}$ at the second node; the buffering capacity is infinite at both nodes. Although traffic may enter the first queue in 'quanta' of positive size, we assume that it leaves the first queue as 'fluid'. In the standard example of packets fed into a communication network, it would mean that during the service of packets in the first queue, the second queue fills at (net) rate $c_{1}-c_{2}$.

Let $\{A(t), t \in \mathbb{R}\}$ be the input process; then the amount of traffic generated in the interval $(s, t]$, where $s<t$ and $s, t \in \mathbb{R}$, is denoted by $A(s, t):=A(t)-A(s)$. We first assume that $\{A(t), t \in \mathbb{R}\}$ has stationary increments; later we focus on the case of stationary independent increments. We denote by $Q_{1}, Q_{2}$ the steady-state workload at the first and second queue, respectively. Analogously, we denote by $Q$ the total steady-state workload in the tandem network. We assume that $\mu:=\mathbb{E}\{A(1)\}<c_{2}$ to ensure that both $Q_{1}$ and $Q_{2}$ are finite a.s. 
In this paper we focus on the probability that the stationary buffer content at the downstream (i.e., second) node exceeds level $u$ :

$$
\mathbb{P}\left(Q_{2}>u\right), \text { for } u>0 .
$$

Following Reich's representation [30] we can write for the first queue, using the fact that $A(\cdot)$ has stationary increments,

$$
Q_{1}={ }_{\mathrm{d}} \sup _{t \geq 0}\left\{A(-t, 0)-c_{1} t\right\}={ }_{\mathrm{d}} \sup _{t \geq 0}\left\{A(t)-c_{1} t\right\} .
$$

Now notice that the following 'reduction property' applies, which was also used in [25]. Consider a single queue fed by the original input process $\{A(t), t \in \mathbb{R}\}$, and drained at the slowest of the service rates $\min \left\{c_{1}, c_{2}\right\}$. Then it is not hard to see that the buffer content of this queue is equal to the total buffer content of our tandem system, see, e.g., Avi-Itzhak [5] and Friedman [18]. Hence, we have for the total workload $Q$ that

$$
Q={ }_{\mathrm{d}} \sup _{t \geq 0}\left\{A(-t, 0)-c_{2} t\right\}={ }_{\mathrm{d}} \sup _{t \geq 0}\left\{A(t)-c_{2} t\right\}
$$

because we assumed that $c_{2}<c_{1}$.

The above observations directly lead to the following representation for $Q_{2}$ :

$$
Q_{2}={ }_{\mathrm{d}} \sup _{t \geq 0}\left\{A(t)-c_{2} t\right\}-\sup _{t \geq 0}\left\{A(t)-c_{1} t\right\} .
$$

Remark 2.1 It is tempting to explicitly impose the assumption that $A(t)$ is increasing in $t$, bearing in mind that $A(t)$ is interpreted as the amount of traffic entering the queue in $[0, t)$. However, there are good reasons to not assume a priori that $A(\cdot)$ is increasing, the most prominent being the following.

Consider the superposition of a number of i.i.d. on-off sources, i.e., sources alternating between sending at some constant, positive rate, and being silent; the corresponding input process is evidently increasing. When the number of sources grows large, however, there is, after some appropriate rescaling, convergence to 'limit processes' which are not necessarily increasing, for instance (fractional) Brownian motion (with drift), or Lévy input models, see e.g. $[15,40]$. This motivates our choice to not restrict ourselves to increasing inputs.

Two additional comments need to be made. In the first place, we mention that in many cases the above-mentioned convergence of traffic aggregates carries over to the stationary buffer-content process. Loosely speaking, the queue fed by the aggregate input process converges to the queue fed by the 'limit process', see, e.g., [10, 14, 15]. Secondly, notice that, when using representation (3), the queue length in the downstream node is necessarily positive. 


\subsection{A representation for the workload distribution}

In this section we prove a fundamental result that simplifies the representation (3) for the queue length in the downstream queue $Q_{2}$. In particular, we show that the set over which both suprema in (3) should be taken can be decomposed into two adjacent intervals. Define $t_{u}:=u /\left(c_{1}-c_{2}\right)$, which can be interpreted as the minimum time it takes for the second queue to exceed level $u$, starting empty.

Proposition 2.2 Let $c_{1}>c_{2}>0$, and let $\{X(t), t \geq 0\}$ be a stochastic process satisfying $\lim _{t \rightarrow \infty}\left\{X(t)-c_{2} t\right\}=-\infty$ a.s. Then, for each $u \geq 0$,

$$
\begin{aligned}
\mathbb{P}\left(\sup _{t \geq 0}\left\{X(t)-c_{2} t\right\}-\sup _{t \geq 0}\left\{X(t)-c_{1} t\right\}>u\right)= \\
\quad \mathbb{P}\left(\sup _{t \in\left[t_{u}, \infty\right)}\left\{X(t)-c_{2} t\right\}-\sup _{t \in\left[0, t_{u}\right]}\left\{X(t)-c_{1} t\right\}>u\right) .
\end{aligned}
$$

Proof We use the short notation $Y_{S}^{(i)}:=\sup _{t \in S}\left\{X(t)-c_{i} t\right\}$, for $i=1,2$ and $S \subseteq \mathbb{R}^{+}$. Define also $t_{i}^{\star}:=\arg \sup _{t \geq 0}\left\{X(t)-c_{i} t\right\}$. We have to prove that

$$
\mathbb{P}\left(Y_{[0, \infty)}^{(2)}-Y_{[0, \infty)}^{(1)}>u\right)=\mathbb{P}\left(Y_{\left[t_{u}, \infty\right)}^{(2)}-Y_{\left[0, t_{u}\right]}^{(1)}>u\right) .
$$

Our proof is in four steps.

- We first prove that $Y_{[0, \infty)}^{(2)}-Y_{[0, \infty)}^{(1)}>u$ implies that $t_{2}^{\star} \geq t_{u}$. To show this, suppose that $t_{2}^{\star}<t_{u}$; then we have that

$$
\begin{aligned}
Y_{[0, \infty)}^{(2)}-Y_{[0, \infty)}^{(1)} & =\sup _{t \in\left[0, t_{u}\right)}\left\{X(t)-c_{2} t-\sup _{s \geq 0}\left\{X(s)-c_{1} s\right\}\right\} \\
& \leq \sup _{t \in\left[0, t_{u}\right)}\left\{X(t)-c_{2} t-\left\{X(t)-c_{1} t\right\}\right\}=u
\end{aligned}
$$

- Because of the first step, we can restrict ourselves to $t_{2}^{\star} \geq t_{u}$. Consequently, we can decompose our probability into

$$
\begin{aligned}
& \mathbb{P}\left(Y_{[0, \infty)}^{(2)}-Y_{[0, \infty)}^{(1)}>u\right)=\mathbb{P}\left(Y_{\left[t_{u}, \infty\right)}^{(2)}-Y_{[0, \infty)}^{(1)}>u\right) \\
& \quad=\mathbb{P}\left(Y_{\left[t_{u}, \infty\right)}^{(2)}-Y_{[0, \infty)}^{(1)}>u ; t_{1}^{\star}>t_{u}\right)+\mathbb{P}\left(Y_{\left[t_{u}, \infty\right)}^{(2)}-Y_{[0, \infty)}^{(1)}>u ; t_{1}^{\star} \leq t_{u}\right)
\end{aligned}
$$

call the latter probabilities $P_{1}$ and $P_{2}$, respectively.

- First consider $P_{1}$. Note that under $t_{1}^{\star}>t_{u}$, it holds that

$$
Y_{\left[t_{u}, \infty\right)}^{(2)}-Y_{[0, \infty)}^{(1)} \geq\left(X\left(t_{1}^{\star}\right)-c_{2} t_{1}^{\star}\right)-\left(X\left(t_{1}^{\star}\right)-c_{1} t_{1}^{\star}\right)>u,
$$


which combined with the fact that

$$
\left\{Y_{\left[t_{u}, \infty\right)}^{(2)}-Y_{[0, \infty)}^{(1)}>u\right\} \subseteq\left\{Y_{\left[t_{u}, \infty\right)}^{(2)}-Y_{\left[0, t_{u}\right]}^{(1)}>u\right\}
$$

implies that

$$
P_{1}=\mathbb{P}\left(Y_{\left[t_{u}, \infty\right)}^{(2)}-Y_{\left[0, t_{u}\right]}^{(1)}>u ; t_{1}^{\star}>t_{u}\right) .
$$

- We trivially have

$$
P_{2}=\mathbb{P}\left(Y_{\left[t_{u}, \infty\right)}^{(2)}-Y_{\left[0, t_{u}\right]}^{(1)}>u ; t_{1}^{\star} \leq t_{u}\right) .
$$

Thus

$$
P_{1}+P_{2}=\mathbb{P}\left(Y_{\left[t_{u}, \infty\right)}^{(2)}-Y_{\left[0, t_{u}\right]}^{(1)}>u\right) .
$$

This completes the proof.

We now return to the class of arrival processes with stationary increments, that was introduced in Section 2.1. For this class the above result enables us to rewrite (3) as follows.

Theorem 2.3 For each $u \geq 0$,

$$
\mathbb{P}\left(Q_{2}>u\right)=\mathbb{P}\left(\sup _{t \in\left[t_{u}, \infty\right)}\left\{A(t)-c_{2} t\right\}-\sup _{t \in\left[0, t_{u}\right]}\left\{A(t)-c_{1} t\right\}>u\right) .
$$

Proof The statement follows immediately by combining (3) with Proposition 2.2.

If the increments of $A(\cdot)$ are also independent, then we get the following representation.

Theorem 2.4 Let $\{A(t), t \in \mathbb{R}\}$ be a stochastic process with stationary independent increments and let $\mu=\mathbb{E} A(1)<c_{2}$. Then for each $u \geq 0$, and $A_{1}(\cdot)$ and $A_{2}(\cdot)$ independent copies of the process $A(\cdot)$,

$$
\mathbb{P}\left(Q_{2}>u\right)=\mathbb{P}\left(\sup _{t \in[0, \infty)}\left\{A_{1}(t)-c_{2} t\right\}>\sup _{t \in\left[0, t_{u}\right]}\left\{-A_{2}(t)+c_{1} t\right\}\right) .
$$

Proof To get the result, use that

$$
\begin{aligned}
& \sup _{t \in\left[t_{u}, \infty\right)}\left\{A(t)-c_{2} t\right\}-\sup _{t \in\left[0, t_{u}\right]}\left\{A(t)-c_{1} t\right\}= \\
& \sup _{t \in\left[t_{u}, \infty\right)}\left\{A(t)-A\left(t_{u}\right)-c_{2}\left(t-t_{u}\right)\right\}-\sup _{t \in\left[0, t_{u}\right]}\left\{A(t)-A\left(t_{u}\right)-c_{1}\left(t-t_{u}\right)\right\}+\left(c_{1}-c_{2}\right) t_{u} .
\end{aligned}
$$

Now the stated follows immediately from the independence and stationarity of the increments of $A(\cdot)$, in conjunction with the fact that $\left(c_{1}-c_{2}\right) t_{u}=u$. 


\section{Distribution of $\mathbb{P}\left(Q_{2}>u\right)$}

This section studies the distribution of $\mathbb{P}\left(Q_{2}>u\right)$. Direct application of the new representation of the downstream queue, as derived in the previous section, yields the Laplace transform $\mathbb{E e}^{-x Q_{2}}$ and the Pollaczek-Khintchine representation of the distribution of $Q_{2}$.

\subsection{Laplace transform}

In this section we assume that $A(\cdot) \equiv\{A(t), t \in \mathbb{R}\}$ is a spectrally positive Lévy process, see, e.g., [35] (thus allowing positive jumps). Hence its Laplace transform is uniquely determined by the Laplace exponent $\kappa(\cdot)$ through

$$
\mathbb{E} \mathrm{e}^{-s A(t)}=\mathrm{e}^{t \kappa(s)},
$$

$s \geq 0$; see for instance [4, Ch. IX.9].

We need the following additional notation:

$$
\begin{aligned}
& S_{1}:=\sup _{t \geq 0}\left\{A_{1}(t)-c_{2} t\right\} ; \\
& S_{2, T}:=\sup _{t \in[0, T]}\left\{-A_{2}(t)+c_{1} t\right\},
\end{aligned}
$$

where $A_{1}(\cdot), A_{2}(\cdot)$ are independent copies of the process $A(\cdot)$. So we can rewrite formula (4) in Theorem 2.4, with $t_{u}:=u /\left(c_{1}-c_{2}\right)$, to

$$
\mathbb{P}\left(Q_{2} \leq u\right)=\mathbb{P}\left(S_{1}-S_{2, t_{u}} \leq 0\right) .
$$

It appears that the function $\theta_{1}(\cdot)$, with

$$
\theta_{1}(s):=\log \left(\mathbb{E} \mathrm{e}^{-s A(1)+s c_{1}}\right)
$$

is of crucial importance in the further analysis; observe that $\theta_{1}(s)=\kappa(s)+c_{1} s$. Additionally let $R_{y}$ denote the first-passage time of level $y: R_{y}:=\inf \left\{t \geq 0:-A_{2}(t)+c_{1} t>y\right\}$, so that

$$
\mathbb{P}\left(S_{2, t_{u}}<y\right)=\mathbb{P}\left(R_{y}>t_{u}\right)
$$

for each $y \geq 0$. We first summarize some useful properties of $\theta_{1}(\cdot)$ and $R_{y}$.

Proposition 3.1 Let $\{A(t), t \in \mathbb{R}\}$ be a spectrally positive Lévy process with Laplace exponent $\kappa(\cdot)$ and $\mu:=\mathbb{E} A(1)<c_{2}$. Then

(i) $\theta_{1}(s)$ is strictly increasing on $s \in[0, \infty)$;

(ii) $\theta_{1}(s) / s \rightarrow c_{1}-\mu$ as $s \downarrow 0$;

(iii) $\mathbb{E}^{-s R_{y}}=\mathrm{e}^{-y \theta_{1}^{-1}(s)}$, for $s \geq 0$ and $y \geq 0$, where $\theta_{1}^{-1}(\cdot)$ is the inverse function of $\theta_{1}(\cdot)$. 
Proof Part (i) follows from the fact that $\theta_{1}^{\prime}(0+)=-\mathbb{E} A(1)+c_{1}=-\mu+c_{1}>0$, in conjunction with the well known property that $\kappa(\cdot)$ is convex. Part (ii) is a consequence of $\theta_{1}(s) / s \rightarrow \theta_{1}^{\prime}(0+)$ as $s \downarrow 0$. Part (iii) is an immediate implication of Sato [35, Theorem 46.3]; see also, e.g., [8, 19].

In the following theorem we obtain an explicit formula for the Laplace transform of the downstream queue $Q_{2}$.

Theorem 3.2 Let $\{A(t), t \in \mathbb{R}\}$ be a spectrally positive Lévy process with Laplace exponent $\kappa(\cdot)$ and $\mu:=\mathbb{E} A(1)<c_{2}$. Then, for each $x>0$,

$$
\mathbb{E}^{-x Q_{2}}=\frac{c_{2}-\mu}{c_{1}-c_{2}} \cdot \frac{\theta_{1}^{-1}\left(x\left(c_{1}-c_{2}\right)\right)}{x-\theta_{1}^{-1}\left(x\left(c_{1}-c_{2}\right)\right)} .
$$

Proof Let $x>0$. In the first step we prove that

$$
\mathbb{E} \mathrm{e}^{-x Q_{2}}=\mathbb{E} \mathrm{e}^{-\theta_{1}^{-1}\left(x\left(c_{1}-c_{2}\right)\right) S_{1}} .
$$

Note that integration by parts yields

$$
\begin{aligned}
\mathbb{E} \mathrm{e}^{-x Q_{2}} & =\int_{0}^{\infty} \mathrm{e}^{-x u} \mathrm{~d} \mathbb{P}\left(Q_{2} \leq u\right)=x \int_{0}^{\infty} \mathrm{e}^{-x u} \mathbb{P}\left(Q_{2} \leq u\right) \mathrm{d} u \\
& =1-x \int_{0}^{\infty} \mathrm{e}^{-x u} \mathbb{P}\left(Q_{2}>u\right) \mathrm{d} u
\end{aligned}
$$

Moreover, due to (5) and (6) we have

$$
\begin{aligned}
\int_{0}^{\infty} \mathrm{e}^{-x u} \mathbb{P}\left(Q_{2}>u\right) \mathrm{d} u & =\int_{0}^{\infty} \mathrm{e}^{-x u} \mathbb{P}\left(S_{1}-S_{2, t_{u}}>0\right) \mathrm{d} u \\
& =\int_{0}^{\infty} \mathrm{e}^{-x u} \int_{0}^{\infty} \mathbb{P}\left(S_{2, t_{u}}<y\right) \mathrm{d} \mathbb{P}\left(S_{1} \leq y\right) \mathrm{d} u \\
& =\int_{0}^{\infty} \mathrm{e}^{-x u} \int_{0}^{\infty} \mathbb{P}\left(R_{y}>t_{u}\right) \mathrm{d} \mathbb{P}\left(S_{1} \leq y\right) \mathrm{d} u .
\end{aligned}
$$

Applying the change of variable $v:=u /\left(c_{1}-c_{2}\right)$, this leads to

$$
\begin{aligned}
\int_{0}^{\infty} & \mathrm{e}^{-x u} \mathbb{P}\left(Q_{2}>u\right) \mathrm{d} u= \\
& =\left(c_{1}-c_{2}\right) \int_{0}^{\infty} \int_{0}^{\infty} \mathrm{e}^{-x\left(c_{1}-c_{2}\right) v} \mathbb{P}\left(R_{y}>v\right) \mathrm{d} v \mathrm{~d} \mathbb{P}\left(S_{1} \leq y\right) \\
& =\left(c_{1}-c_{2}\right) \int_{0}^{\infty}\left(\frac{1}{x\left(c_{1}-c_{2}\right)}-\int_{0}^{\infty} \mathrm{e}^{-x\left(c_{1}-c_{2}\right) v} \mathbb{P}\left(R_{y} \leq v\right) \mathrm{d} v\right) \mathrm{d} \mathbb{P}\left(S_{1} \leq y\right) \\
& =\frac{1}{x}\left(1-\int_{0}^{\infty} \int_{0}^{\infty} \mathrm{e}^{-x\left(c_{1}-c_{2}\right) v} \mathrm{~d} \mathbb{P}\left(R_{y} \leq v\right) \mathrm{d} \mathbb{P}\left(S_{1} \leq y\right)\right) \\
& =\frac{1}{x}\left(1-\int_{0}^{\infty} \mathrm{e}^{-y \theta_{1}^{-1}\left(x\left(c_{1}-c_{2}\right)\right)} \mathrm{d} \mathbb{P}\left(S_{1} \leq y\right)\right) \\
& =\frac{1}{x}\left(1-\mathbb{E}^{-\theta_{1}^{-1}\left(x\left(c_{1}-c_{2}\right)\right) S_{1}}\right),
\end{aligned}
$$


where (9) is due to part (iii) of Proposition 3.1. Now (7) follows directly from combining (8) with (10).

In order to complete the proof note that, following Zolotarev's result for spectrally positive Lévy processes [42], for $x>0$

$$
\mathbb{E} \mathrm{e}^{-x S_{1}}=\frac{c_{2}-\mu}{\theta_{2}(x)} x
$$

where $\theta_{2}(s):=\log \left(\mathbb{E} \mathrm{e}^{-s A(1)+s c_{2}}\right)$; observe that $\theta_{2}(s)=\kappa(s)+c_{2} s=\theta_{1}(s)+\left(c_{2}-c_{1}\right) s$. Combining (7) with (11) leads to

$$
\begin{aligned}
\mathbb{E}^{-x Q_{2}} & =\frac{\left(c_{2}-\mu\right) \theta_{1}^{-1}\left(x\left(c_{1}-c_{2}\right)\right)}{\theta_{2}\left(\theta_{1}^{-1}\left(x\left(c_{1}-c_{2}\right)\right)\right)} \\
& =\frac{c_{2}-\mu}{c_{1}-c_{2}} \frac{\theta_{1}^{-1}\left(x\left(c_{1}-c_{2}\right)\right)}{x-\theta_{1}^{-1}\left(x\left(c_{1}-c_{2}\right)\right)} .
\end{aligned}
$$

This completes the proof.

Remark 3.3 Theorem 3.2 can be considered as an analogue of the result of Zolotarev [42]; there the Laplace transform of $\mathbb{P}\left(Q_{1}<u\right)$ was given for $A(\cdot)$ being a spectrally positive Lévy process, whereas here we present the corresponding result for $\mathbb{P}\left(Q_{2}<u\right)$.

Remark 3.4 Recall that $Q$ denotes the (steady-state) of the total workload. Since $S_{1}={ }_{\mathrm{d}} Q$, it follows that (7) is equivalent to

$$
\mathbb{E}^{-x Q_{2}}=\mathbb{E} \mathrm{e}^{-\theta_{1}^{-1}\left(x\left(c_{1}-c_{2}\right)\right) Q},
$$

for $x \geq 0$. This relation plays a crucial role in Proposition 5.1 .

Remark 3.5 We note that for special cases the above theorem can be derived in alternative ways. Consider for instance the case of compound Poisson input. We assume that $A(t)=\sum_{i=1}^{N(t)} X_{i}$, where $N(\cdot)$ is a Poisson process with rate $\lambda$. The random variables $X_{i}$ are i.i.d., nonnegative and independent of $N(\cdot)$. We denote by $X$ the generic random variable, and by $F(\cdot)$ its distribution function. Under the above notation, we say that $A(\cdot)$ is a compound Poisson process, characterized by $(\lambda, F)$. Let $f:=\mathbb{E} X$.

Observe that, in the tandem setting, the downstream queue is fed by an on-off process: during on-periods, which are distributed as the busy period of the first queue, the downstream queue fills at rate $c_{1}-c_{2}$, whereas during off-periods, which have an exponential distribution (with mean $\lambda^{-1}$ ), it drains at rate $c_{2}$. Assume for ease $\mu=\lambda f<1=c_{2}<2=$ $c_{1}$ (the analysis can be extended straightforwardly to the case of general service rates). Under this choice, our formula for $\mathbb{E} \mathrm{e}^{-x Q_{2}}$ reduces to

$$
\mathbb{E} \mathrm{e}^{-x Q_{2}}=(1-\mu) \cdot \frac{\theta_{1}^{-1}(x)}{x-\theta_{1}^{-1}(x)} .
$$


Denoting by $P$ the busy period of the first queue, and $\pi(s):=\mathbb{E} \mathrm{e}^{-s P}$, and $\xi(s):=\mathbb{E} \mathrm{e}^{-s X}$, then $[2$, Eq. (3)] gives

$$
\mathbb{E}^{-x Q_{2}}=\frac{1-\lambda \mathbb{E} P}{1+\lambda \mathbb{E} P} \cdot \frac{x+\lambda-\lambda \pi(x)}{x-\lambda+\lambda \pi(x)} .
$$

It is now left to check that the above two formulas coincide. As the input rate of the first queue equals the output rate of the first queue

$$
\mu=\lambda f=\frac{\mathbb{E} P}{1 / \lambda+\mathbb{E} P} \cdot 2,
$$

we obtain that $1-\mu=(1-\lambda \mathbb{E} P) /(1+\lambda \mathbb{E} P)$. Hence it is left to show that

$$
\frac{\theta_{1}^{-1}(x)}{x-\theta_{1}^{-1}(x)}=\frac{x+\lambda-\lambda \pi(x)}{x-\lambda+\lambda \pi(x)},
$$

or, equivalently, $2 \theta_{1}^{-1}(x)=x+\lambda-\lambda \pi(x)$, or

$$
\theta_{1}\left(\frac{x}{2}+\frac{\lambda}{2}-\frac{\lambda}{2} \pi(x)\right)=x
$$

By definition we have $\theta_{1}(x)=-\lambda+\lambda \xi(x)+2 x$. Also, we have the well-known fixed-point equation for the busy period of the $M / G / 1$ queue (with service rate 2 ):

$$
\pi(x)=\xi\left(\frac{x}{2}+\frac{\lambda}{2}-\frac{\lambda}{2} \pi(x)\right)
$$

Hence we obtain (13):

$$
\theta_{1}\left(\frac{x}{2}+\frac{\lambda}{2}-\frac{\lambda}{2} \pi(x)\right)=-\lambda+\lambda \xi\left(\frac{x}{2}+\frac{\lambda}{2}-\frac{\lambda}{2} \pi(x)\right)+x+\lambda-\lambda \pi(x)=x,
$$

as desired. Related results can be found in $[24,36]$.

\subsection{Pollaczek-Khintchine representation}

Theorem 3.2 enables us to determine Pollaczek-Khintchine representation for the distribution function of $Q_{2}$. Define $\widetilde{R}_{y}:=\left(c_{1}-c_{2}\right) R_{y}$ and observe that, due to Proposition 3.1, $\left\{\widetilde{R}_{y}: y \geq 0\right\}$ is a subordinator with $\mathbb{E} \mathrm{e}^{-x R_{y}}=\mathrm{e}^{-y \vartheta(x)}$, where $\vartheta(x):=\theta_{1}^{-1}\left(\left(c_{1}-c_{2}\right) x\right)$, for $y \geq 0$ and $x \geq 0$. Recalling (ii) in Proposition 3.1, we have

$$
\varrho:=\lim _{x \downarrow 0} \frac{\vartheta(x)}{x}=\frac{c_{1}-c_{2}}{c_{1}-\mu}
$$

with $\varrho \in(0,1)$. 
Thus, following e.g. Takács [38, Eq. (23)], define a distribution function $H(\cdot)$ such that $H(x)=0$ for $x<0$ and its Laplace transform $\hat{\ell}_{H}(s)$ is of the form

$$
\hat{\ell}_{H}(x)=\int_{0}^{\infty} \mathrm{e}^{-x v} \mathrm{~d} H(v)=\frac{\vartheta(x)}{\varrho x}
$$

for $x \geq 0$.

Theorem 3.6 Let $\{A(t), t \in \mathbb{R}\}$ be a spectrally positive Lévy process with Laplace exponent $\kappa(\cdot)$ and $\mu:=\mathbb{E} A(1)<c_{2}$. Then

$$
\mathbb{P}\left(Q_{2} \leq u\right)=(1-\varrho) \sum_{i=1}^{\infty} \varrho^{i-1} H^{\star i}(u) .
$$

Proof Application of Theorem 3.2 immediately yields

$$
\begin{aligned}
\mathbb{E}^{-x Q_{2}} & =\frac{c_{2}-\mu}{c_{1}-c_{2}} \frac{\vartheta(x)}{x-\vartheta(x)}=\frac{c_{2}-\mu}{c_{1}-c_{2}} \frac{\varrho \frac{\vartheta(x)}{\varrho x}}{1-\varrho \frac{\vartheta(x)}{\varrho x}} \\
& =\frac{c_{2}-\mu}{c_{1}-c_{2}} \sum_{i=1}^{\infty}\left(\varrho \hat{\ell}_{H}(x)\right)^{i}=(1-\varrho) \sum_{i=1}^{\infty} \varrho^{i-1} \hat{\ell}_{H}^{i}(x) .
\end{aligned}
$$

This implies the stated immediately.

Remark 3.7 Theorem 3.6 is related to Theorem 4.1 in [25], where it was assumed that the input process is a compound Poisson.

Remark 3.8 The distribution $H(\cdot)$ has a natural representation in the language of the Lévy measure associated with $\left\{\widetilde{R}_{y}: y \geq 0\right\}$. Indeed, since $\left\{\widetilde{R}_{y}: y \geq 0\right\}$ is a subordinator, then, following e.g. [7, Section 3.1, Eq. (3)], the Lévy-Khintchine representation of the Laplace exponent $\vartheta(\cdot)$ has the following form:

$$
\vartheta(x)=\mathrm{d} x+x \int_{0}^{\infty} \mathrm{e}^{-x s} \bar{\Pi}(s) \mathrm{d} s,
$$

where $\mathrm{d}$ is the drift coefficient and $\bar{\Pi}(s)=\Pi((s, \infty))$ is the tail of the Lévy measure. Then

$$
\hat{\ell}_{H}(x)=\frac{\mathrm{d}}{\varrho}+\frac{1}{\varrho} \int_{0}^{\infty} \mathrm{e}^{-x s} \bar{\Pi}(s) \mathrm{d} s .
$$

Hence

$$
H(t)=\frac{\mathrm{d}}{\varrho}+\frac{1}{\varrho} \int_{0}^{t} \bar{\Pi}(s) \mathrm{d} s
$$

for all $t \geq 0$. Observe also that $\varrho=\mathrm{d}+\int_{0}^{\infty} \bar{\Pi}(s) \mathrm{d} s$. 


\section{Examples: light-tailed case}

In this section we apply results of previous sections to two special cases. The first focuses on Brownian input, the second on Poisson input; hence, in all cases the increments are independent, and consequently Equation (4) can be used. Before analyzing particular examples, let us first identify a 'dichotomy' in the distribution of $Q_{2}$.

\subsection{A dichotomy}

If $\kappa(s)$ exists for negative $s$, the Lévy process could be called 'light-tailed', as the tail of the distribution of $A(1)$ decays exponentially or faster. In this section we use the results of the previous section to further investigate this light-tailed case, eventually leading to an interesting dichotomy. Throughout this subsection, we tacitly assume that the Lévy input is spectrally positive.

Bearing in mind part (iii) in Proposition 3.1, it is seen that by analytic continuation, Theorem 3.2 holds for all $x$ for which $\theta_{1}^{-1}\left(x\left(c_{1}-c_{2}\right)\right)$ is well defined. For light-tailed Lévy processes, this means that we could pick any $x$ larger than $x_{1}:=\left(c_{1}-c_{2}\right)^{-1} \min _{y \in \mathbb{R}} \theta_{1}(y)$ (use that $\theta_{1}(\cdot)$ is convex). Theorem 3.2 implies that if, in addition, $x>\theta_{1}^{-1}\left(x\left(c_{1}-c_{2}\right)\right)$, we have that $\mathbb{E} \mathrm{e}^{-x Q_{2}}$ is finite, and hence 'Chernoff' implies that

$$
\mathbb{P}\left(Q_{2}>u\right) \leq M e^{x u},
$$

for some finite constant $M$ - see also [1, Lemma 3.2]. Let $x_{2}$ solve $x_{2}=\theta_{1}^{-1}\left(x_{2}\left(c_{1}-c_{2}\right)\right)$, or, equivalently, $\theta_{2}(x)=0$; if there is no solution, then take the infimum of all $x$ such that $\theta_{2}(x) \leq 0$ (this $x_{2}$ is necessarily negative). The above arguments immediately lead to the following result, yielding the tightest $x$ for which (15) holds.

Proposition 4.1 If $x_{2}>\arg \min _{y} \theta_{1}(y)$ then Inequality (15) holds (for some $M<\infty$ ) for all $x>x_{1}$; else Inequality (15) holds (for some $M<\infty$ ) for all $x>x_{2}$.

Assuming that $\kappa(\cdot)$ is differentiable, define $c_{1}^{\star}:=-\kappa^{\prime}\left(x_{2}\right)$; notice that $x_{2}$ depends on $c_{2}$ only (not on $c_{1}$ ), and consequently also $c_{1}^{\star}$ depends on $c_{2}$ only. The next result indicates that $c_{1}^{\star}$ can be viewed as a critical service rate, demarcating two regimes.

Proposition 4.2 If $c_{1}<c_{1}^{\star}$ then Inequality (15) holds (for some $M<\infty$ ) for all $x>x_{1}$; else Inequality (15) holds (for some $M<\infty$ ) for all $x>x_{2}$.

Proof Due to the convexity of $\theta_{1}(\cdot)$, requiring $x_{2}>\arg \inf _{y} \theta_{1}(y)$ is equivalent to requiring $\theta_{1}^{\prime}\left(x_{2}\right)>0$. This implies the stated immediately. 
The above indicates that there are in fact two regimes: there is essentially different behavior for $c_{1}>c_{1}^{\star}$ and $c_{1} \leq c_{1}^{\star}$. It is noted that in the first case the exponential bound (with decay rate $x_{2}$ ) coincides with the traditional Kingman-type bound of a single queue with service rate $c_{2}$. We return to this phenomenon later in this paper; there we also give an interpretation of the two regimes.

\subsection{Tandem with Brownian motion input}

In this subsection we analyze representation (4) for the case where $A(\cdot)=B(\cdot)$, a standard Brownian motion (with zero drift). It appears that an explicit formula can be derived for $\mathbb{P}\left(Q_{2}>u\right)$. Let $\Psi(\cdot)$ denote the tail distribution of a standard Normal random variable $N$ : $\Psi(x)=\mathbb{P}(N>x)$.

Theorem 4.3 Let $A(\cdot)$ be a standard Brownian motion. Then, for each $u \geq 0$,

$$
\mathbb{P}\left(Q_{2}>u\right)=\frac{c_{1}-2 c_{2}}{c_{1}-c_{2}} \mathrm{e}^{-2 c_{2} u}\left(1-\Psi\left(\frac{c_{1}-2 c_{2}}{\sqrt{c_{1}-c_{2}}} \sqrt{u}\right)\right)+\frac{c_{1}}{c_{1}-c_{2}} \Psi\left(\frac{c_{1}}{\sqrt{c_{1}-c_{2}}} \sqrt{u}\right) .
$$

Proof Following Theorem 2.4 we have

$$
\mathbb{P}\left(Q_{2}>u\right)=\mathbb{P}\left(\sup _{t \in[0, \infty)}\left\{B_{1}(t)-c_{2} t\right\}>\sup _{t \in\left(0, t_{u}\right]}\left\{B_{2}(t)+c_{1} t\right\}\right),
$$

where $B_{1}(\cdot), B_{2}(\cdot)$ are independent standard Brownian motions. Using the well-known fact that, for each $x \geq 0$,

$$
\mathbb{P}\left(\sup _{t \in[0, \infty)}\left\{B_{1}(t)-c_{2} t\right\}>x\right)=\mathrm{e}^{-2 c_{2} x}
$$

and (see, e.g., Baxter and Donsker [6])

$$
\begin{aligned}
\rho(x) & :=\frac{\mathrm{d}}{\mathrm{d} x} \mathbb{P}\left(\sup _{t \in\left(0, t_{u}\right]}\left\{B_{2}(t)+c_{1} t\right\} \leq x\right) \\
& =\sqrt{\frac{2}{\pi t_{u}}} \exp \left(-\frac{\left(x-c_{1} t_{u}\right)^{2}}{2 t_{u}}\right)-2 c_{1} \mathrm{e}^{2 c_{1} x} \Psi\left(\frac{x+c_{1} t_{u}}{\sqrt{t_{u}}}\right),
\end{aligned}
$$

we get two integrals, that we will calculate separately:

$$
\mathbb{P}\left(Q_{2}>u\right)=\int_{0}^{\infty} \mathrm{e}^{-2 c_{2} x} \rho(x) \mathrm{d} x=I_{1}(u)-I_{2}(u),
$$

where

$$
\begin{aligned}
& I_{1}(u):=\int_{0}^{\infty} \mathrm{e}^{-2 c_{2} x} \sqrt{\frac{2}{\pi t_{u}}} \exp \left(-\frac{\left(x-c_{1} t_{u}\right)^{2}}{2 t_{u}}\right) \mathrm{d} x \\
& I_{2}(u):=\int_{0}^{\infty} \mathrm{e}^{-2 c_{2} x} 2 c_{1} \mathrm{e}^{2 c_{1} x} \Psi\left(\frac{x+c_{1} t_{u}}{\sqrt{t_{u}}}\right) \mathrm{d} x .
\end{aligned}
$$


Integral $I_{1}(u)$ is evaluated as follows:

$$
\begin{aligned}
I_{1}(u) & =2 \int_{0}^{\infty} \frac{1}{\sqrt{2 \pi t_{u}}} \exp \left(-\frac{x^{2}-2 c_{1} t_{u} x+c_{1}^{2} t_{u}^{2}+4 c_{2} x t_{u}}{2 t_{u}}\right) \mathrm{d} x \\
& =2 \mathrm{e}^{-c_{2}\left(c_{1}-c_{2}\right) t_{u}} \int_{0}^{\infty} \frac{1}{\sqrt{2 \pi t_{u}}} \exp \left(-\frac{\left(x-\left(c_{1}-2 c_{2}\right) t_{u}\right)^{2}}{2 t_{u}}\right) \mathrm{d} x \\
& =2 \mathrm{e}^{-2 c_{2} u}\left(1-\Psi\left(\frac{c_{1}-2 c_{2}}{\sqrt{c_{1}-c_{2}}} \sqrt{u}\right)\right) .
\end{aligned}
$$

For $I_{2}(u)$ we find

$$
\begin{aligned}
I_{2}(u) & =\int_{0}^{\infty} 2 c_{1} \mathrm{e}^{2 x\left(c_{1}-c_{2}\right)} \Psi\left(\frac{x+c_{1} t_{u}}{\sqrt{t_{u}}}\right) \mathrm{d} x \\
& =\frac{c_{1}}{c_{1}-c_{2}} \int_{0}^{\infty} 2\left(c_{1}-c_{2}\right) \mathrm{e}^{2 x\left(c_{1}-c_{2}\right)} \Psi\left(\frac{x+c_{1} t_{u}}{\sqrt{t_{u}}}\right) \mathrm{d} x .
\end{aligned}
$$

Now, integration by parts gives that $I_{2}(u)=c_{1} \tilde{I}_{2}(u) /\left(c_{1}-c_{2}\right)$, with

$$
\begin{aligned}
\tilde{I}_{2}(u)= & -\Psi\left(c_{1} \sqrt{t_{u}}\right)+\int_{0}^{\infty} \mathrm{e}^{2 x\left(c_{1}-c_{2}\right)} \frac{1}{\sqrt{2 \pi t_{u}}} \exp \left(-\frac{\left(x+c_{1} t_{u}\right)^{2}}{2 t_{u}}\right) \mathrm{d} x \\
= & -\Psi\left(c_{1} \sqrt{t_{u}}\right)+\exp \left(-\frac{c_{1}^{2} t_{u}}{2}+\frac{\left(c_{1}-2 c_{2}\right)^{2} t_{u}}{2}\right) \times \\
& \quad \int_{0}^{\infty} \frac{1}{\sqrt{2 \pi t_{u}}} \exp \left(-\frac{x^{2}-2 x t_{u}\left(c_{1}-2 c_{2}\right)+\left(c_{1}-2 c_{2}\right)^{2} t_{u}^{2}}{2 t_{u}}\right) \mathrm{d} x \\
= & -\Psi\left(c_{1} \sqrt{t_{u}}\right)+\mathrm{e}^{-c_{2}\left(2 c_{1}-2 c_{2}\right) t_{u}} \int_{0}^{\infty} \frac{1}{\sqrt{2 \pi t_{u}}} \exp \left(-\frac{\left(x-\left(c_{1}-2 c_{2}\right) t_{u}\right)^{2}}{2 t_{u}}\right) \mathrm{d} x \\
= & -\Psi\left(c_{1} \sqrt{t_{u}}\right)+\mathrm{e}^{-2 c_{2} u}\left(1-\Psi\left(\frac{c_{1}-2 c_{2}}{\sqrt{c_{1}-c_{2}}} \sqrt{u}\right)\right) .
\end{aligned}
$$

Combining (16) and (17) yields the desired result.

The next corollary finds the exact asymptotics of the buffer content distribution in the second node, by applying the general result of Theorem 4.3 .

Corollary 4.4 Let $A(\cdot)$ be a standard Brownian motion.

(i) If $c_{1}>2 c_{2}$, then

$$
\mathbb{P}\left(Q_{2}>u\right)=\frac{c_{1}-2 c_{2}}{c_{1}-c_{2}} \mathrm{e}^{-2 c_{2} u}(1+o(1)) \text { as } u \rightarrow \infty .
$$

(ii) If $c_{1}=2 c_{2}$, then

$$
\mathbb{P}\left(Q_{2}>u\right)=\frac{1}{\sqrt{2 \pi c_{2}}} \frac{1}{\sqrt{u}} \mathrm{e}^{-2 c_{2} u}(1+o(1)) \text { as } u \rightarrow \infty .
$$


(iii) If $c_{1}<2 c_{2}$, then

$$
\mathbb{P}\left(Q_{2}>u\right)=\frac{1}{\sqrt{2 \pi}}\left(\frac{c_{1}-c_{2}}{u}\right)^{3 / 2} \frac{4 c_{2}}{c_{1}^{2}\left(c_{1}-2 c_{2}\right)^{2}} \exp \left(-\frac{c_{1}^{2}}{2\left(c_{1}-c_{2}\right)} u\right)(1+o(1)) \text { as } u \rightarrow \infty .
$$

Proof Let $J_{1}(u)$ and $J_{2}(u)$ be defined as

$$
J_{1}(u):=\frac{c_{1}-2 c_{2}}{c_{1}-c_{2}} \mathrm{e}^{-2 c_{2} u}\left(1-\Psi\left(\frac{c_{1}-2 c_{2}}{\sqrt{c_{1}-c_{2}}} \sqrt{u}\right)\right)
$$

and

$$
J_{2}(u):=\frac{c_{1}}{c_{1}-c_{2}} \Psi\left(\frac{c_{1}}{\sqrt{c_{1}-c_{2}}} \sqrt{u}\right)
$$

recall from Theorem 4.3 that $\mathbb{P}\left(Q_{2}>u\right)=J_{1}(u)+J_{2}(u)$. We also recall the standard 'first order' asymptotic result

$$
\Psi(u)=\frac{1}{\sqrt{2 \pi}} \frac{1}{u} \mathrm{e}^{-u^{2} / 2}(1+o(1)),
$$

and 'second order' result

$$
\frac{1}{\sqrt{2 \pi}} \frac{1}{u} \mathrm{e}^{-u^{2} / 2}-\Psi(u)=\frac{1}{\sqrt{2 \pi}} \frac{1}{u^{3}} \mathrm{e}^{-u^{2} / 2}(1+o(1)) .
$$

We now consider the three cases separately.

(i) $c_{1}>2 c_{2}$. Then

$$
\lim _{u \rightarrow \infty} \frac{\log J_{1}(u)}{\log J_{2}(u)}=\frac{c_{1}^{2}}{4 c_{2}\left(c_{1}-c_{2}\right)}=\frac{\left(c_{1} / c_{2}\right)^{2}}{4\left(c_{1} / c_{2}-1\right)}>1,
$$

due to $x^{2}>4(x-1)>0$ for $x>2$. The stated follows after applying (18).

(ii) $c_{1}=2 c_{2}$. Then $J_{1}(u)=0$, and the stated follows from (18).

(iii) $c_{1}<2 c_{2}$. In this case, remarkably, both $-J_{1}(u)$ and $J_{2}(u)$ equal, as $u \rightarrow \infty$,

$$
\sqrt{\frac{1}{c_{1}-c_{2}}} \frac{1}{\sqrt{2 \pi}} \frac{1}{\sqrt{u}} \exp \left(-\frac{c_{1}}{2\left(c_{1}-c_{2}\right)} u\right)(1+o(1))
$$

due to (18). As a consequence, we have to rely on the more precise asymptotics (19). After tedious computations we derive the stated. 
We see that there are two regimes: with $c_{1}^{\star}:=2 c_{2}$, there is essentially different behavior for $c_{1}>c_{1}^{\star}$ and $c_{1} \leq c_{1}^{\star}$; notice that, in the terminology of Section 4.1, we indeed have that $c_{1}^{\star}=-\kappa^{\prime}\left(x_{2}\right)$ (with $\kappa(s)=\frac{1}{2} s^{2}$ and $x_{2}=-2 c_{2}$ ). In the first case, upon overflow in the second queue the first queue remains essentially empty, and consequently the largebuffer asymptotics resemble those of a single queue with service rate $c_{2}$. In the second case, the first queue will be exactly 'balanced', as traffic is generated at about a rate of $c_{1}$, during $t_{u}$ units of time.

\subsection{Tandem with Poisson input}

Now we assume that $A_{i}(t)$, with $i=1,2$, is Poisson distributed with mean $\lambda t$ (for some $\left.\lambda \in\left(0, c_{2}\right)\right)$. We first determine

$$
\mathbb{P}\left(\sup _{t \in[0, \infty)}\left\{A_{1}(t)-c_{2} t\right\}>x\right) \text { and } \mathbb{P}\left(\sup _{t \in\left[0, t_{u}\right]}\left\{-A_{2}(t)+c_{1} t\right\} \geq x\right) .
$$

Consider the first of these probabilities. For instance using the Beneš approach, see, e.g., Roberts et al. [31, Eq. (15.1.3)], with $\rho:=\lambda / c_{2}$,

$$
\zeta(x):=\mathbb{P}\left(\sup _{t \in[0, \infty)}\left\{A_{1}(t)-c_{2} t\right\}>x\right)=(1-\rho) \sum_{n=\lceil x\rceil}^{\infty} \frac{(\rho(n-x))^{n}}{n !} \mathrm{e}^{-\rho(n-x)} .
$$

Now consider the second probability. Evidently,

$$
\eta(x):=\mathbb{P}\left(\sup _{t \in\left[0, t_{u}\right]}\left\{-A_{2}(t)+c_{1} t\right\} \geq x\right)=\mathbb{P}\left(\exists t \in\left[0, t_{u}\right]: A_{2}(t) \leq c_{1} t-x\right) .
$$

This expression can be computed by conditioning on the value of $A_{2}\left(t_{u}\right)$, as follows:

$$
\begin{aligned}
& \mathbb{P}\left(\exists t \in\left[0, t_{u}\right]: A_{2}(t) \leq c_{1} t-x\right)=\mathbb{P}\left(A_{2}\left(t_{u}\right) \leq c_{1} t_{u}-x\right) \\
& \quad+\sum_{i=\left\lceil c_{1} t_{u}-x\right\rceil}^{\infty} \mathbb{P}\left(\exists t \in\left[0, t_{u}\right]: A_{2}(t) \leq c_{1} t-x \mid A_{2}\left(t_{u}\right)=i\right) \mathbb{P}\left(A_{2}\left(t_{u}\right)=i\right) .
\end{aligned}
$$

Obviously,

$$
\mathbb{P}\left(A_{2}\left(t_{u}\right) \leq c_{1} t_{u}-x\right)=\sum_{i=0}^{\left\lfloor c_{1} t_{u}-x\right\rfloor} \frac{\left(\lambda t_{u}\right)^{i}}{i !} \mathrm{e}^{-\lambda t_{u}} ; \quad \mathbb{P}\left(A_{2}\left(t_{u}\right)=i\right)=\frac{\left(\lambda t_{u}\right)^{i}}{i !} \mathrm{e}^{-\lambda t_{u}} .
$$

Finally, focus on $\mathbb{P}\left(\exists t \in\left[0, t_{u}\right]: A_{2}(t) \leq c_{1} t-x \mid A_{2}\left(t_{u}\right)=i\right)$. Reversing time, it is easily seen that this probability coincides with

$$
\mathbb{P}\left(\exists t \in\left[0, t_{u}\right]: A_{2}(t) \geq i+x-c_{1} t_{u}+c_{1} t \mid A_{2}\left(t_{u}\right)=i\right) .
$$


Given $A_{2}\left(t_{u}\right)=i$, these $i$ arrivals are uniformly distributed on $\left[0, t_{u}\right]$. Hence, probability (21) equals $p\left(i+x-c_{1} t_{u}, c_{1} \mid i, t_{u}\right)$, where $p(x, c \mid N, D)$ is the overflow probability over level $x$ in a queue with $N$ periodic inputs (with period $D$ ) and service rate $c$ (which is sometimes referred to as the $N \cdot \mathrm{D} / \mathrm{D} / 1$ queue). An explicit expression for $p(x, c \mid N, D)$ is given in the following lemma.

Lemma 4.5 For $x \in[0, N]$ and $N<x+c \cdot D$, it holds that

$$
p(x, c \mid N, D)=\sum_{n \in \mathbb{N}: n \in(x, N]} \mathbb{B i n}\left(n \mid N, \frac{n-x}{c \cdot D}\right) \cdot \frac{c \cdot D-N+x}{c \cdot D-n+x},
$$

with

$$
\mathbb{B i n}(n \mid N, p):=\left(\begin{array}{c}
N \\
n
\end{array}\right) p^{n}(1-p)^{N-n} .
$$

If $N \geq x+c \cdot D$, then $p(x, c \mid N, D)=0$.

Proof See Humblet et al. [21, Section III.C], and Roberts and Virtamo [32].

This leads to the following expression:

$$
\begin{aligned}
& \eta(x)=\mathbb{P}\left(\sup _{t \in\left[0, t_{u}\right]}\left\{-A_{2}(t)+c_{1} t\right\}>x\right)= \\
& \sum_{i=0}^{\left\lfloor c_{1} t_{u}-x\right\rfloor} \frac{\left(\lambda t_{u}\right)^{i}}{i !} \mathrm{e}^{-\lambda t_{u}}+\sum_{i=\left\lceil c_{1} t_{u}-x\right\rceil}^{\infty} p\left(i+x-c_{1} t_{u}, c_{1} \mid i, t_{u}\right) \frac{\left(\lambda t_{u}\right)^{i}}{i !} \mathrm{e}^{-\lambda t_{u}} .
\end{aligned}
$$

Theorem 4.6 Let $A(\cdot)$ be a Poisson process with rate $\lambda \in\left(0, c_{2}\right)$. Then, for each $u \geq 0$,

$$
\mathbb{P}\left(Q_{2}>u\right)=-\int_{[0, \infty)} \zeta(x) \mathrm{d} \eta(x),
$$

where $\zeta(\cdot)$ and $\eta(\cdot)$ are given by $(20)$ and $(22)$, respectively.

Remark 4.7 As in Corollary 4.4 one could pursue the exact distribution of $Q_{2}$, as in Theorem 4.6, to find its large-buffer asymptotics. However, this turns out to be a non-trivial task, due to the complicated structure of (23).

An alternative is to use a procedure that is similar to the one pursued in [1] for the priority queue. First realize that the output process of the first queue consists of independent onand off-periods. The off-periods are obviously exponential (with mean $\lambda^{-1}$ ), whereas the on-periods correspond to the duration of the busy period, say $B$, in the first queue. The 
asymptotics of the busy period are known since Cox and Smith [13, Section 5.6], and are of the type

$$
\mathbb{P}(B>u)=\alpha u^{-3 / 2} \mathrm{e}^{-\beta u}(1+o(1)),
$$

for positive numbers $\alpha, \beta$, and $u \rightarrow \infty$. Standard results for the large-buffer asymptotics of queues fed by a single on-off source now indicate that, as in the Brownian case, there are two regimes, separated by a critical service rate.

The critical service rate can be found as follows. Let $\theta^{\star}>0$ solve $\log \mathbb{E} \mathrm{e}^{\theta A(t)}=c_{2} \theta t$, or, equivalently, $\lambda\left(\mathrm{e}^{\theta}-1\right)=c_{2} \theta$, and define $\lambda^{\star}:=\lambda \mathrm{e}^{\theta^{\star}}$. If $c_{1}>c_{1}^{\star}:=\lambda^{\star}$, then the first queue is essentially transparent, and the large-buffer asymptotics are purely exponen-

tial: $\alpha_{+} \mathrm{e}^{-\beta_{+} u}$, with $\beta_{+}:=\theta^{\star}$. For $c_{1}<c_{1}^{\star}$, the large-buffer asymptotics are of the form $\alpha_{-} u^{-3 / 2} \mathrm{e}^{-\beta_{-} u}$; in this case

$$
\beta_{-}=\left(\frac{1}{c_{1}-c_{2}}\right)\left(c_{1} \log \frac{c_{1}}{\lambda}-c_{1}+\lambda\right) \text {. }
$$

We recognize in (24) the Kullback-Leibler distance of a Poisson $\left(c_{1}\right)$ random variable with respect to a Poisson $(\lambda)$ random variable. Indeed, (24) can be interpreted as the decay rate of the Poisson source generating traffic at rate $c_{1}$ during $t_{u}$ units of time, thus building up an amount of traffic $u$ in the downstream queue.

Remark 4.8 The approach presented in this subsection is not restricted to Poisson input of batches of deterministic (unit) size. Let us for instance consider the situation of a Poisson $(\lambda)$ stream of nonnegative i.i.d. batches $\left(A_{i}\right)_{i \in \mathbb{N}}$. It turns out that Theorem 1 of Takács [39, p. 24] is quite useful here. To make this theorem directly applicable, assume for the moment that time is slotted (i.e., the number of batches arriving per slot has a Poisson distribution with mean $\lambda$ ), $c_{1}=1$, and that the batch sizes are i.i.d. and take on nonnegative integral values.

We can repeat the above argument. Now $\zeta(\cdot)$ is the overflow probability in the corresponding M/G/1 queue, which is explicitly given by the Pollaczek-Khintchine formula, see for instance [4, Thm. 5.7, Eq. (5.5)]. Also $\eta(\cdot)$ follows easily: we can use exactly the same procedure as above, by first conditioning on the value of $A_{2}\left(t_{u}\right)$ - notice that the per-slot arrivals are interchangeable random variables, such that the counterpart of probability (21) follows immediately from [39, Thm. 1, p. 24].

\section{Examples: heavy-tailed case}

In this section we analyze the asymptotics of the tail distribution of $Q_{2}$ in a case when $A(\cdot)$ possesses a heavy-tailed structure. 
Before we focus on particular examples, we need the following proposition, which relates the asymptotic behavior of $\mathbb{P}\left(Q_{2}>u\right)$ with $\mathbb{P}(Q>u)$; recall that $Q$ represents the total workload.

Proposition 5.1 Let $v \in(0,1)$ and

$$
\mathbb{P}(Q>u)=u^{-v} \ell(u)(1+o(1))
$$

as $u \rightarrow \infty$, where $\ell(\cdot)$ is slowly varying at $\infty$. Then

$$
\mathbb{P}\left(Q_{2}>u\right)=\varrho^{v} u^{-v} \ell(u)(1+o(1))
$$

as $u \rightarrow \infty$.

Proof Assume that, as $u \rightarrow \infty, \mathbb{P}(Q>u)=u^{-v} \ell(u)(1+o(1))$ for $v \in(0,1)$. Then, following Lemma 2.2 in [9], we have

$$
\mathbb{E} \mathrm{e}^{-x Q}-1=\Gamma(1-v) x^{v} \ell\left(\frac{1}{x}\right)(1+o(1))
$$

as $x \rightarrow 0$. Thus, the use of (12) leads to

$$
\begin{aligned}
\mathbb{E}^{-x Q_{2}}-1 & =\mathbb{E}^{-\theta_{1}^{-1}\left(x\left(c_{1}-c_{2}\right)\right) Q}-1= \\
& =\Gamma(1-v) \theta_{1}^{-1}\left(x\left(c_{1}-c_{2}\right)\right)^{v} \ell\left(\frac{1}{\theta_{1}^{-1}\left(x\left(c_{1}-c_{2}\right)\right)}\right)(1+o(1)) \\
& =\Gamma(1-v)\left(\frac{\theta_{1}^{-1}\left(x\left(c_{1}-c_{2}\right)\right)}{x}\right)^{v} x^{v} \ell\left(\frac{x}{\theta_{1}^{-1}\left(x\left(c_{1}-c_{2}\right)\right)} \frac{1}{x}\right)(1+o(1)) \\
& =\Gamma(1-v) \varrho^{v} x^{v} \ell\left(\frac{1}{x}\right)(1+o(1)),
\end{aligned}
$$

as $x \rightarrow 0$, since $\lim _{x \downarrow 0} \theta_{1}^{-1}\left(x\left(c_{1}-c_{2}\right)\right) / x=\varrho$. Thus, again, the use of [9, Lemma 2.2] completes the proof.

In the following proposition we give the exact asymptotics of the tail distribution of $Q_{2}$ in a case when $H(\cdot)$ belongs to the class of subexponential distributions.

Proposition 5.2 Let $\{A(t), t \in \mathbb{R}\}$ be a spectrally positive Lévy process with $\mu:=\mathbb{E} A(1)<c_{2}$. If $H(\cdot)$ defined in (14) is subexponential, then

$$
\mathbb{P}\left(Q_{2}>u\right)=\frac{c_{1}-\mu}{c_{2}-\mu} \bar{H}(u)(1+o(1))
$$

as $u \rightarrow \infty$. 
Proof From Theorem 3.6 we have that for each $u \geq 0$

$$
\begin{aligned}
\mathbb{P}\left(Q_{2} \leq u\right) & =(1-\varrho) \sum_{i=1}^{\infty} \varrho^{i-1} H^{\star i}(u) \\
& =(1-\varrho) \sum_{i=0}^{\infty} \varrho^{i}\left(H^{\star i} \star H\right)(u)=(K \star H)(u),
\end{aligned}
$$

where $K(u)=(1-\varrho) \sum_{i=0}^{\infty} \varrho^{i} H^{\star i}(u)$ and $\varrho$, as before, defined as $\left(c_{1}-c_{2}\right) /\left(c_{1}-\mu\right)$. Now, following e.g. Embrechts et al. [16],

$$
\bar{K}(u)=\frac{\varrho}{1-\varrho} \bar{H}(u)(1+o(1))
$$

as $u \rightarrow \infty$; the bars indicate complementary distribution functions. Thus using the convolution closure property of the class of subexponential random variables; see e.g. Embrechts \& Goldie [17] or Cline [12], we conclude that

$$
\begin{aligned}
\mathbb{P}\left(Q_{2}>u\right) & =\overline{K \star H}(u)=\left(\frac{\varrho}{1-\varrho}+1\right) \bar{H}(u)(1+o(1)) \\
& =\frac{1}{1-\varrho} \bar{H}(u)(1+o(1))=\frac{c_{1}-\mu}{c_{2}-\mu} \bar{H}(u)(1+o(1)) .
\end{aligned}
$$

This completes the proof.

\subsection{Tandem with $\alpha$-stable Lévy input}

In this subsection we focus on the case of $\alpha$-stable Lévy input. Let $X_{\alpha, \sigma, \beta}(\cdot)$ denote an $\alpha$-stable Lévy motion with scale parameter $\sigma$ and skewness parameter $\beta$, see, e.g., [34]. We analyze $\mathbb{P}\left(Q_{2}>u\right)$ for $A(\cdot)$ an $\alpha$-stable Lévy motion with $\alpha \in(1,2), \sigma=1$ and $\beta \in(-1,1]$. for the general case of $\beta \in(-1,1]$ we present the generic asymptotic upper and lower bounds for $\mathbb{P}\left(Q_{2}>u\right)$ in the large-buffer regime, i.e., $u \rightarrow \infty$. Moreover, if $\beta=1$, then the application of Proposition 5.1 provides us with the exact asymptotics of $\mathbb{P}\left(Q_{2}>u\right)$.

In the rest of this section we use the notation

$$
\begin{aligned}
& B_{1}(\alpha, \beta):=\frac{\Gamma(1+\alpha)}{\pi} \sqrt{1+\beta^{2} \tan ^{2}(\pi \alpha / 2)} \sin \left(\frac{\pi \alpha}{2}+\arctan (\beta \tan (\pi \alpha / 2))\right) \\
& B_{2}(\alpha, \beta):=\frac{1+\beta}{2} \frac{\alpha-1}{\Gamma(2-\alpha) \cos (\pi(\alpha-2) / 2)} .
\end{aligned}
$$

Theorem 5.3 Let $A(t)=X_{\alpha, 1, \beta}(t)$ with $\alpha \in(1,2)$ and $\beta \in(-1,1]$. Then

(i) as $u \rightarrow \infty$,

$$
\mathbb{P}\left(Q_{2}>u\right) \leq\left(\frac{B_{1}(\alpha, \beta)}{c_{2} \alpha(\alpha-1)}+\frac{B_{2}(\alpha, \beta)}{c_{1}}\right)\left(\frac{c_{1}}{c_{1}-c_{2}}\right)^{1-\alpha} u^{1-\alpha}(1+o(1))
$$


(ii) as $u \rightarrow \infty$,

$$
\mathbb{P}\left(Q_{2}>u\right) \geq \frac{B_{1}(\alpha, \beta)}{c_{2} \alpha(\alpha-1)}\left(\frac{c_{1}}{c_{1}-c_{2}}\right)^{1-\alpha} u^{1-\alpha}(1+o(1)) .
$$

The following lemma from [29] - see also [20, Prop. 3.7], where the substitution $\beta:=-\beta$ matches our notation - is needed in our analysis.

Lemma 5.4 Let $\alpha \in(1,2), \beta \in(-1,1]$ and $c>0$. Then

$$
\mathbb{P}\left(\sup _{t \in[0, \infty)}\left\{X_{\alpha, 1, \beta}(t)-c t\right\}>u\right)=\frac{B_{1}(\alpha, \beta)}{c \alpha(\alpha-1)} u^{1-\alpha}(1+o(1)),
$$

as $u \rightarrow \infty$.

Proof of Theorem 5.3 Let $X_{\alpha, 1, \beta}(\cdot)$ and $Y_{\alpha, 1, \beta}(\cdot)$ be mutually independent $\alpha$-stable Lévy motions and recall that, as before, $t_{u}=u /\left(c_{1}-c_{2}\right)$.

Ad. (i): following Theorem 2.4 we have that

$$
\left.\mathbb{P}\left(Q_{2}>u\right) \leq \mathbb{P}\left(\sup _{t \in[0, \infty)}\left\{X_{\alpha, 1, \beta}(t)-c_{2} t\right\}+Y_{\alpha, 1, \beta}\left(t_{u}\right)>c_{1} t_{u}\right\}\right) .
$$

Moreover, using self-similarity of $Y_{\alpha, 1, \beta}(\cdot)$ and following [34, Property 1.2.15],

$$
\begin{aligned}
\mathbb{P}\left(Y_{\alpha, 1, \beta}\left(t_{u}\right)>c_{1} t_{u}\right) & =\mathbb{P}\left(Y_{\alpha, 1, \beta}(1)>c_{1} t_{u}^{(\alpha-1) / \alpha}\right) \\
& =B_{2}(\alpha, \beta) c_{1}^{-\alpha} t_{u}^{1-\alpha}(1+o(1)),
\end{aligned}
$$

as $u \rightarrow \infty$.

Combining (26) with Lemma 5.4 and using that both asymptotics are regularly varying at infinity, we immediately obtain

$$
\begin{aligned}
\mathbb{P}\left(Q_{2}>u\right) & \leq\left(\frac{B_{1}(\alpha, \beta)}{c_{2} \alpha(\alpha-1)} c_{1}^{1-\alpha}+B_{2}(\alpha, \beta) c_{1}^{-\alpha}\right) t_{u}^{1-\alpha}(1+o(1)) \\
& =\left(\frac{B_{1}(\alpha, \beta)}{c_{2} \alpha(\alpha-1)}+\frac{B_{2}(\alpha, \beta)}{c_{1}}\right)\left(\frac{c_{1}}{c_{1}-c_{2}}\right)^{1-\alpha} u^{1-\alpha}(1+o(1)),
\end{aligned}
$$

as $u \rightarrow \infty$, which completes the proof of (i).

Ad. (ii): Following Theorem 2.4 we have that

$$
\mathbb{P}\left(Q_{2}>u\right)=\mathbb{P}\left(\sup _{t \in[0, \infty)}\left\{X_{\alpha, 1, \beta}(t)-c_{2} t\right\}>\sup _{t \in\left[0, t_{u}\right]}\left\{-Y_{\alpha, 1, \beta}(t)+c_{1} t\right\}\right) .
$$


Hence

$$
\begin{aligned}
\mathbb{P}\left(Q_{2}>u\right) & \geq \mathbb{P}\left(\sup _{t \in[0, \infty)}\left\{X_{\alpha, 1, \beta}(t)-c_{2} t\right\}-\sup _{t \in\left[0, t_{u}\right]}\left\{-Y_{\alpha, 1, \beta}(t)\right\}>c_{1} t_{u}\right) \\
& =\mathbb{P}\left(\sup _{t \in[0, \infty)}\left\{X_{\alpha, 1, \beta}(t)-c_{2} t\right\}>c_{1} t_{u}\right)(1+o(1)) \\
& =\frac{B_{1}(\alpha, \beta)}{c_{2} \alpha(\alpha-1)}\left(\frac{c_{1}}{c_{1}-c_{2}}\right)^{1-\alpha} u^{1-\alpha}(1+o(1)),
\end{aligned}
$$

as $u \rightarrow \infty$, where (27) follows from [28, Lemma 6.3] combined with the fact that, due to Lemma 5.4, $\sup _{t \in[0, \infty)}\left\{X_{\alpha, 1, \beta}(t)-c_{2} t\right\}$ belongs to the class of subexponential random variables. Equation (28) straightforwardly follows from Lemma 5.4. This completes the proof.

In the following theorem we analyze an important special case of the $\alpha$-stable Lévy input process where $\beta=1$. The importance of this class of input processes stems, e.g., from $[27,40]$, where it was proved that $X_{\alpha, 1,1}(\cdot)(\alpha \in(1,2))$ may be used as an approximation for an appropriately scaled superposition of many on-off input processes.

Theorem 5.5 Let $A(\cdot)=X_{\alpha, 1,1}(\cdot)$ with $\alpha \in(1,2)$. Then as $u \rightarrow \infty$,

$$
\mathbb{P}\left(Q_{2}>u\right)=\frac{1}{\Gamma(2-\alpha) \cos (\pi(\alpha-2) / 2)} \frac{1}{c_{2}}\left(\frac{c_{1}}{c_{1}-c_{2}}\right)^{1-\alpha} u^{1-\alpha}(1+o(1)) .
$$

Proof The combination of Proposition 5.1 with Lemma 5.4 gives that

$$
\mathbb{P}\left(Q_{2}>u\right)=\frac{B_{1}(\alpha, \beta)}{c_{2} \alpha(\alpha-1)}\left(\frac{c_{1}}{c_{1}-c_{2}}\right)^{1-\alpha} u^{1-\alpha}(1+o(1)) .
$$

Routine calculation, as in [20, Ch. 3], yields that

$$
B_{1}(\alpha, 1)=\frac{\alpha(\alpha-1)}{\Gamma(2-\alpha) \cos (\pi(\alpha-2) / 2)},
$$

which completes the proof.

Remark 5.6 Observe that the exact asymptotic presented in Theorem 5.5 matches the lower bound given in Theorem 5.3. We conjecture that this observation extends to the whole range of parameters $\beta \in(-1,1]$. 


\subsection{Tandem with compound Poisson input, with regularly varying jumps}

Consider again the case of compound Poisson $(\lambda, F)$ input. In the following theorem we present the exact asymptotics of the tail distribution of $Q_{2}$ under the assumption that $\bar{F}(\cdot)$ is regularly varying at $\infty$.

Theorem 5.7 Let $A(\cdot)$ correspond to a compound Poisson process $(\lambda, F)$. Suppose that

$$
1-F(x)=x^{-\alpha} \ell(x),
$$

where $\alpha \in(1,2), \ell(\cdot)$ is slowly varying at $\infty$ and $c_{2}>\lambda \mathbb{E} X$. Then

$$
\mathbb{P}\left(Q_{2}>u\right)=\frac{\lambda}{c_{2}-\lambda \mathbb{E} X}\left(\frac{c_{1}-\lambda \mathbb{E} X}{c_{1}-c_{2}}\right)^{1-\alpha} \frac{1}{\alpha-1} u^{1-\alpha} \ell(u)(1+o(1))
$$

as $u \rightarrow \infty$.

Proof Observe that $\mathbb{E} A(t)=\lambda \mathbb{E} X t$. Following e.g. [3, Thm. 2.1] we have that

$$
\mathbb{P}(Q>u)=\frac{\lambda \mathbb{E} X}{c_{2}-\lambda \mathbb{E} X} \frac{1}{\mathbb{E} X}\left(\int_{u}^{\infty}(1-F(x)) \mathrm{d} x\right)(1+o(1))
$$

as $u \rightarrow \infty$. Thus, using Karamata's theorem, we have that

$$
\mathbb{P}(Q>u)=\frac{\lambda}{c_{2}-\lambda \mathbb{E} X} \frac{1}{\alpha-1} u^{1-\alpha} \ell(u)(1+o(1))
$$

as $u \rightarrow \infty$. Hence the stated follows by application of Proposition 5.1.

\section{Acknowledgment}

The first and second author would like to thank the Mittag-Leffler Institute, Djursholm, Sweden, for hospitality. Part of the work was done while the first and third author were also at CWI. The first author was partially supported by University of Wrocław under Grant 2529/W/IM/2004, and by KBN Grant No 1 P03A 03128 (2005-2007). Pascal Lieshout (CWI) is thanked for providing useful remarks concerning Corollary 4.4.

\section{References}

[1] Abate, J. and Whitt, W. (1997). Asymptotics for M/G/1 low-priority waiting-time tail probabilities. Queueing Syst. 25, 173-233.

[2] Adan, I. and Resing, J. (1996). A note on a fluid queue driven by an $M / M / 1$ queue. Queueing Syst. 22, 171-174. 
[3] Asmussen, S. (2000). Ruin Probabilities. World Scientific, London.

[4] Asmussen, S. (2003). Applied Probability and Queues. Springer, New York.

[5] Avi-Itzhak, B. (1965). A sequence of service stations with arbitrary input and regular service times. Man. Sci. 11, 565-571.

[6] Baxter, G. and Donsker, M.D. (1957). On the distribution of the supremum functional for the processes with stationary independent increments. Trans. Amer. Math. Soc. 85, 73-87.

[7] Bertoin, J. (1996). Léry Processes, Cambridge University Press, Cambridge.

[8] Bingham, N. (1975). Fluctuation theory in continuous time. Adv. Appl. Prob. 7, 705766.

[9] Boxma, O. and Dumas, V. (1998). The busy period in the fluid queue. Perf. Eval. Review 26, 100-110.

[10] Cao, J. and Ramanan, K. (2002). A Poisson limit for buffer overflow probabilities. Proceedings IEEE INFOCOM, New York, NY, 994-1003.

[11] Chang, C.-S., P. Heidelberger, S. Juneja, and Shahabuddin, P. (1994). Effective bandwidth and fast simulation of ATM intree networks. Perf. Eval. 20, 45-65.

[12] Cline, D.B.H (1986). Convolution tails, product tails and domains of attraction. Probab. Theory and Related Fields 72, 529-557.

[13] Cox, D. and Smith, W. (1961). Queues. Methuen, London.

[14] Dębicki, K. and Mandjes, M. (2004). Traffic with an fBm limit: Convergence of the stationary workload process. Queueing Syst. 46, 113-127.

[15] Dębicki, K. and Palmowski, Z. (1999). On-off fluid models in heavy traffic environment. Queueing Syst. 33, 327-338.

[16] Embrechts, P., Goldie, C.M. and Veraverbeke, N. (1979). Subexponentiality and infinite divisibility. Z. Wahrsch. Verw. Geb. 49, 335-347.

[17] Embrechts, P. and Goldie, C.M. (1982). On convolution tails. Stoch. Proc. Appl. 13, 263-268.

[18] Friedman, H. (1965). Reduction methods for tandem queueing systems. Oper. Res. 13, 121-131. 
[19] Fristedt, B. (1974). Sample functions of stochastic processes with stationary independent increments. Advances in Probability 3, 241-396. Dekker, New York NY.

[20] Furrer, H. (1997). Risk theory and heavy-tailed Lévy processes. Ph.D. dissertation. Available at http://www.math.ethz.ch/ ${ }^{\sim h j f u r r e r / . ~}$

[21] Humblet, P., Bhargava, A. and Hluchyj, M. (1993). Ballot theorems applied to the transient analysis of $\mathrm{nD} / \mathrm{D} / 1$ queues. IEEE/ACM Trans. Netw. 1, 81-95.

[22] Kaj, I. and Taqqu, M. (2004). Convergence to fractional Brownian motion and to the Telecom process: the integral representation approach.

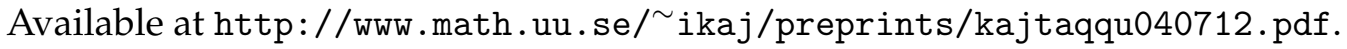

[23] Kella, O. (1993). Parallel and tandem fluid networks with dependent Lévy inputs. Ann. Appl. Prob. 3, 682-695.

[24] Kella, O. (2001). Markov-modulated feedforward fluid networks. Queueing Syst. 37, 141-161.

[25] Kella, O. and Whitt, W. (1992). A tandem fluid network with Lévy input. In: Queueing and related models, U. Bhat and I. Basawa (eds). Clarendon Press, Oxford.

[26] Mandjes, M. and van Uitert, M. (2005). Sample-path large deviations for tandem and priority queues with Gaussian inputs. Ann. Appl. Prob. 15, 1193-1226.

[27] Mikosch, T., Resnick, S., Rootzén, H. and Stegeman, A. (2002). Is network traffic traffic approximated by stable Lévy motion or fractional Brownian motion? Ann. Appl. Prob. 12, 23-68.

[28] Pitman, E.J.G. (1980). Subexponential distribution functions. J. Austral. Math.Soc. (Series A) 29, 337-347.

[29] Port, S. (1989). Stable processes with drift on the line. Trans. Am. Math. Soc. 313, 805841.

[30] Reich, E. (1958). On the integrodifferential equation of Takács I. Ann. Math. Stat. 29, 563-570.

[31] Roberts, J., Mocci, U. and Virtamo, J. (1996). Broadband network teletraffic. Final report of action COST 242. Springer, Berlin.

[32] Roberts, J. and Virtamo, J. (1991). The superposition of periodic cell arrival streams in an ATM multiplexer. IEEE Trans. Comm. 39, 298-303. 
[33] Rubin, I. (1974). Path delays in communication networks. Appl. Math. and Opt., 1, 193-221.

[34] Samorodnitsky, G. and Taqqu, M. (1994). Stable non-Gaussian Random Processes, Chapman and Hall, London.

[35] Sato, K. (1999). Léry processes and infinitely divisible distributions, Cambridge University Press, Cambridge.

[36] Scheinhardt, W. and Zwart, B. (2002). A tandem fluid queue with gradual input. Prob. Eng. Inf. Sci. 16, 29-45.

[37] Shalmon, M. and Kaplan, M. (1984). A tandem network of queues with deterministic service and intermediate arrivals. Oper. Res. 32, 753-773.

[38] Takács, L. (1965). On the distribution of the supremum of stochastic processes with exchangeable increments. Trans. Am. Math. Soc. 119, 367-379.

[39] Takács, L. (1967). Combinatorial methods in the theory of stochastic processes. Wiley, New York NY.

[40] Taqqu, M., Willinger, W. and Sherman, R. (1997). Proof of a fundamental result in self-similar traffic modeling. Comp. Comm. Rev. 27, 5-23.

[41] Takagi, H. (1991). Queueing Analysis. A foundation of Performance Evaluation. Vol. I: Vacation and Priority Systems, Part 1. North-Holland, Amsterdam.

[42] Zolotarev, V.M. (1964). The first passage time of a level and the behaviour at infinity for a class of processes with independent increments. Th. Prob. Appl. 9, 653-661. 